\title{
Histoplasmosis diseminada con síndrome hemofagocítico asociado con infección por HTLV-1. Reporte de caso
}

\begin{abstract}
Disseminated histoplasmosis with hemophagocytic syndrome associated with HTLV-1 infection. Case report
Ronald Ventosilla ${ }^{1, a}$, Daniel del Carpio ${ }^{1, b}$

RESUMEN

Síndrome hemofagocítico (SH) es un cuadro sistémico severo usualmente fatal, reportado como causa secundaria en adultos. Histoplasmosis ha sido descrita como una de las causas, siempre en pacientes con inmunosupresión. Se presenta el caso de un paciente varón natural de Huánuco con historia de tos y expectoración de dos meses e infiltrado pulmonar intersticial y parenquimal basal derecho con adenopatía mediastinal. Ingresó con fiebre, pancitopenia, alteración de pruebas hepáticas y pruebas de coagulación que cumplía criterios de SH. En el aspirado de médula ósea se observaron inclusiones intracitoplasmáticas en los macrófagos compatibles con Histoplasma capsulatum. La serología para HTLV-1 fue positiva por lo que se analiza el rol de este virus como agente inmunosupresor que condiciona SH secundario a histoplasmosis diseminada.
\end{abstract}

PALABRAS CLAVE: Linfohistiocitosis hemofagocítica, histoplasmosis, virus 1 linfotrópico T humano. (Fuente: DeCS BIREME).

\section{SUMMARY}

The hemophagocytic syndrome (HS) is a severe systemic illness ultimately fatal associated with underlying conditions. Histoplasmosis has been associated with HS in immunosuppressed patients. We present the case of a male patient from Huanuco with a 2-month history of productive cough and presence of interstitial pulmonary infiltrates, and consolidation in the right lower lobe associated with mediastinal lymphadenopathies on the chest $\mathrm{x}$-ray. The patient presented with fever, pancytopenia and abnormalities in the liver and coagulation tests fulfilling criteria for HS. A bone marrow aspiration showed intracytoplasmic structures within the macrophages compatible with Histoplasma capsulatum. Serology for HTLV-1 was positive. We analyzed the role of HTLV-1 inducing immunosuppression leading to the development of disseminated histoplasmosis and HS.

KEYWORDS: Lymphohistiocytosis, hemophagocytic, hystoplasmosis, human T -lymphotropic virus 1. (Source: MeSH NLM).

\footnotetext{
Hospital Edgardo Rebagliati Martins, EsSalud. Lima, Perú.

Médico Residente de Oncología Médica;

Médico Asistente de Hematología
} 


\section{INTRODUCCIÓN}

El Síndrome hemofagocítico (SH) conocido también como linfohistiocitosis hemofagocitica comprende un grupo heterogéneo de desórdenes de cuadro similar a la sepsis combinado con hemofagocitosis, pancitopenia, hiperferritinemia e hipercitoquinemia, que resulta frecuentemente en falla orgánica múltiple (1). Desde la primera descripción en 1939 por Scott y Robb Smith (2), se han descrito mejor sus mecanismos, clasificándolos como primarios o forma genética, predominante en población pediátrica y el secundario, asociado a infecciones, procesos autoinmunes o neoplasias(1,3), y con criterios diagnósticos propuestos por la Histiocyte Society (4). Los mecanismos fisiopatológicos en la etiología primaria, involucran defectos genéticos que conllevan a un estado de inmunodeficiencia, activación inmune significativa o inmunopatología anormal (5). Los defectos genéticos permiten la presencia de una serie de proteínas mutadas (perforina, proteina asociada al SLAM, proteína inhibidora de apoptosis ligada a X) que desencadenan un estado inmune deficiente. Se desarrollan también a anormalidades genéticas que afectan la degranulación de macrófagos $(5,6)$.

En la forma secundaria de SH existe una o varias entidades que desencadenarían el síndrome (Tabla 1) (7), sin embargo, se debe tener en cuenta que un paciente con una causa secundaria podría tener también una mutación genética de base (6).

Entre las infecciones que pueden desencadenar $\mathrm{SH}$ se describen bacterianas, fúngicas y parasitarias. La infección por HIV sola o con otras infecciones concurrentes son causa frecuente de SH $(8,9)$, también

Tabla 1. Enfermedades asociadas a síndrome hemofagocítico.

\section{SINDROME HEMOFAGOCÍTICO PRIMARIO}

- Síndrome hemofagocítico Familiar Tipo 1-4

- Deficiencias inmunes hereditarias

- Síndrome de Griscelli tipe 2

- Síndrome de Chiedak-Higashi

- Síndrome linfoproliferativo ligado a X

- Síndrome de Hermansky-Pudlak tipe 2

- Síndrome proliferativo autoinmune

\section{SINDROME HEMOFAGOCÍTICO SECUNDARIO}

\section{Infecciones}

- Viral (Herpes, HIV, hepatitis, etc)

- Bacterianas (Micobacteria, Micoplasma, Chlamydia)

- Hongos (Aspergillus, Cándida, Histoplasma)

- Parásitos (Falciparum)

\section{Enfermedad Autoinmune}

- Lupus eritematoso sistémico

- Artritis Reumatoide

- Enfermedad de Still

- Poliarteritis nodosa

- Enfermedad de Sjögren

- Enfermedad mista del tejido conectivo

- Esclerodermia

\section{Malignidad}

- Linfomas células B y T Natural-Killer

- Leucemia

- Quimioterapia

Modificado de Van Koeveringe MP, Brouwer RE.(7). 
infecciones por Citomegalovirus, criptococosis, tuberculosis, toxoplasmosis, varicela, Pneumocystis jirovecci, y complejo MAV (Micobacterium avium) (8).

Histoplasmosis es una causa de SH (10), casi siempre asociada a estado de inmunosupresión (infección por HIV, linfoma u otras neoplasias, corticoterapia crónica, estado pos trasplante) (8), produciendo un cuadro de histoplasmosis diseminada aguda con recuento de CD4 $<200 / \mathrm{ml}$. Su diagnóstico se realiza por hemocultivo o mielocultivo positivos a Histoplasma, detección del antígeno en orina o sangre, o visión directa por tinción de plata con metenamina Gomori $(8,11)$.

Se reporta un caso de histoplasmosis diseminada con síndrome hemofagocítico en un paciente con infección por HTLV-1, por tratarse de una asociación inusual con esta última entidad.

\section{PRESENTACIÓN DEL CASO}

Varón de 76 años natural de Huánuco y procedente de Lima, de profesión docente y ocupación comerciante ambulatorio de golosinas. Tenía antecedente de prostatectomía por hiperplasia benigna a los 55 años, enfermedad pulmonar intersticial desde los 56 años sin tratamiento y cáncer de canal a los 66 años con controles en el servicio de Oncología del hospital hasta tres meses antes de su ingreso y sin evidencia de enfermedad.

Tenía historia de cinco meses de astenia, hiporexia y tos escasa sin fiebre. Dos meses antes de ingreso la tos era seca y se tornó vespertina, y dos semanas

Tabla 2. Evolución de exámenes auxiliares.

\begin{tabular}{lrrrrrrr}
\hline & Día 1 & Día 4 & Día 5 & Día 6 & Día 8 & Día 10 & Día 11 \\
\hline Glucosa (mg/dl) & 92 & & 113 & 130 & 54 & $60 / 39 *$ & 149 \\
Urea (mg/dl) & 46 & & 55 & 69 & 66 & 90 & 135 \\
Creatinina (mg/dl) & 0,46 & & 1,04 & 1,01 & 1,23 & 2,2 & 3,23 \\
TGO (UI/l) & 811 & 1879 & 1375 & 792 & 546 & 426 & 288 \\
TGP (UI/l) & 197 & 344 & 300 & 177 & 108 & 67 & 45 \\
Lactato Deshidrogenasa (mg/dl) & 8601 & 13583 & 14400 & 12908 & 11252 & 9533 & 5523 \\
Fosfatasa alcalina (UI/l) & 618 & 895 & 715 & 682 & 708 & 596 & 400 \\
Bilirrubina Directa (mg/dl) & 1,34 & 2,65 & 3,42 & 4,01 & 5,4 & 5,38 & 6,85 \\
Bilirrubina Total (mg/dl) & 1,6 & 3,09 & 3,61 & 4,43 & 6,09 & 6,35 & 8,1 \\
Hemoglobina (g/dl) & 12,6 & 12,1 & 11 & 9,8 & 10,2 & 11,6 & 10,9 \\
Leucocitos (x mm ${ }^{3}$ ) & 2150 & 1890 & 1450 & 1610 & 2220 & 4550 & 9190 \\
Neutrófilos (x mm ${ }^{3}$ ) & 1410 & 560 & 660 & 1000 & 1470 & 3380 & 7850 \\
Linfocitos (x mm $\left.{ }^{3}\right)$ & 620 & 1030 & 660 & 530 & 620 & 920 & 1030 \\
Plaquetas (x mm $\left.{ }^{3}\right)$ & 25400 & 41000 & 46000 & 56000 & 62000 & 75000 & 35000 \\
Monocitos (x mm ${ }^{3}$ ) & 80 & 190 & 100 & 70 & 90 & 160 & 260 \\
Tiempo de protrombina (s) & & & 21 & 21,09 & 19,73 & 20,87 & 16,09 \\
Tiempo de tromboplastina Parcial (s) & & & 62 & 59,72 & 81,42 & 79,1 & 64,8 \\
Internacional Normalized Ratio (INR) & & & 1,79 & & & & 1,36 \\
Fibrinógeno (mg/dl) & & & 102 & 184 & 102 & & 134 \\
\hline
\end{tabular}

* glicemia en el mismo día

TGO: Transaminasa glutámico-oxalacética

TGP: Transaminasa glutámico-pirúvica 
antes de ingreso se agregó expectoración blanquecina y sensación de alza térmica, acudiendo a médico particular quien indicó ciprofloxacino y metamizol, no presentando mejoría.

Al examen en el servicio de emergencia se encontró PA 100/60; FC: $100 \mathrm{x}^{\prime}$; FR: $22 \mathrm{x}$; ; en aparente regular estado general, deshidratado, buen estado de nutrición, mucosa oral seca, no adenopatías, no edemas, subcrépitantes en mitad superior del hemitórax derecho, hígado palpable a $2 \mathrm{~cm}$ debajo del reborde costal, bazo no palpable; el resto del examen fue no contributorio.

Los exámenes de laboratorio mostraron pancitopenia, elevación de pruebas hepáticas con patrón hepatocelular y tiempos de coagulación prolongados (Tabla 2). La prueba HTLV-1 fue reactiva. No se obtuvo serología para HIV por fallecimiento del paciente.

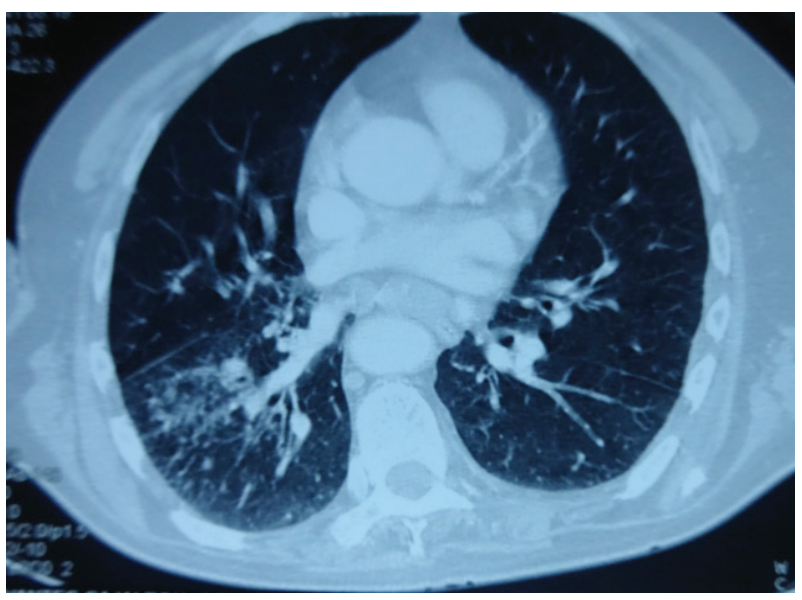

Figura 1. Tomografía de tórax, muestra infiltrado parenquimal en la base del pulmón derecho.

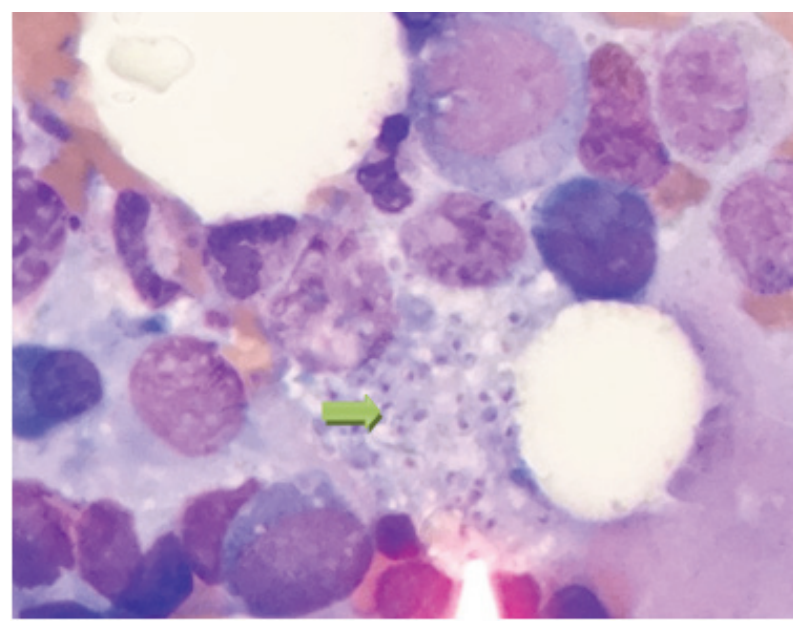

Figura 2. Aspirado de médula ósea, muestra formas redondeadas compatibles con Histoplasma (Flecha).
La ecografía abdominal no reveló tumoraciones, dilatación de vía biliar, liquido libre ni hidronefrosis. Al paciente le habían realizado TAC de tórax tres meses antes del ingreso el que mostraba infiltrado intersticial pulmonar basal derecho y adenopatía mediastinal cuyo tamaño oscilaba entre 20 y $25 \mathrm{~mm}$ (Figura 1).

Inicialmente recibió ceftriaxona y fue hospitalizado en el Servicio de Oncología, presentó fiebre (38,5$39^{\circ} \mathrm{C}$ ), hipoactividad y tendencia al sueño, se cambió la terapia a imipenem y dos días después se agregó vancomicina.

Persistió con pancitopenia y empeoró la función hepática y las pruebas de coagulación (Tabla 1); por esta razón, se agregó a la terapia plasma fresco, vitamina $\mathrm{K}$ y crioprecipitado. Cursó con hipoglicemia (glucosa $54 \mathrm{mg} / \mathrm{dl}$ ) recurrente a pesar de recibir dextrosa al 33\% y al 10\% IV. Desarrolló lesiones dérmicas máculo-papulares diseminadas en tronco y cara por lo que se agregó fluconazol.

Se realizó aspirado de médula ósea el cual mostró serie eritroide y mieloide reactiva, blastos $<1 \%$. Megacariocitos normales. Se observó incremento de histiocitos con abundantes figuras de hemofagocitosis. Se observó imágenes fagocitadas compatibles con Histoplasma capsulatum (Figura 2). En sangre periférica se observó neutrófilos con abundantes granulaciones toxicas y en algunas se observaban estructuras compatibles con Histoplasma capsulatum. Se agregó Anfotericina B al no mostrar mejoría; sin embargo, evolucionó tórpidamente desarrollando falla renal aguda no oligúrica deterioro del nivel de sensorio y con criterios de falla orgánica múltiple, falleció el día siguiente del inicio de anfotericina $\mathrm{B}$.

\section{DISCUSIÓN}

SH asociado a histoplasmosis ocurre en el contexto de histoplasmosis diseminada aguda la cual ocurre en pacientes inmunocomprometidos (11). Sánchez (8), reportó 18 casos de histoplasmosis diseminada con $\mathrm{SH}, 12$ en pacientes con VIH, dos post-trasplantados, uno con enfermedad de Crohn y uno con leucemia linfática crónica. Histoplasmosis diseminada aguda, suele aparecer como consecuencia de una reactivación de una forma crónica de la enfermedad como se ha reportado previamente (12).

El paciente tenía fiebre, adenopatía mediastinal, infiltrados pulmonares, hepato-esplenomegalia, 
rash maculo papular e infiltración de médula ósea, compatibles con histoplasmosis diseminada aguda. El antecedente epidemiológico de nacimiento en Huánuco más la presencia de infiltrado pulmonar y adenopatía, previos al cuadro agudo, son compatibles con infección crónica por Histoplasma, reportada como una de las formas clínicas (13). El diagnóstico de SH se estableció según los criterios propuestos por la Histiocyte Society; el paciente cumplía con seis de los 8 propuestos: Fiebre, esplenomegalia, citopenias (por lo menos 2 líneas celulares), hemofagocitosis, hipofibrinogenemia $(150 \mathrm{mg} / \mathrm{dl})$ y no evidencia de malignidad.

Creemos que el estado de portador de HTLV1 fue el factor que condicionó la inmunosupresión y a su vez su paso a un cuadro reagudizado de histoplasmosis y hemofagocitosis. Los hemocultivos fueron negativos pero el aspirado de médula ósea con formas de Histoplasma fagocitadas por macrófagos son compatibles con SH secundario. El paciente tuvo baciloscopía para TBC negativa desde tres meses antes del ingreso como durante su hospitalización.

HTLV-1 es un virus endémico en nuestro país y de mayor prevalencia en zonas alto andinas del sur del Perú (14). Gotuzzo y col (15), en un reporte nacional reciente encontraron una prevalencia de $2 \%$ en tres provincias de Ayacucho. Esta entidad ha sido descrita que incrementa el riesgo de varias enfermedades infecciosas e inflamatorias, entre ellas, strongiloidiasis y tuberculosis (16), y sobre todo se ha reportado que la infección de HTLV-1 empeora el curso clínico de dichas enfermedades (17).

Aunque los casos asociados a histoplasmosis son escasos (14), la presencia de una infección dual en este caso, aportaría a la hipótesis de la respuesta anormal del hospedero producido por HTLV-1 que provocaría una mayor severidad de la infección micótica (18), como la observada en el paciente.

A diferencia de lo que ocurre en HIV que destruye los linfocitos CD4, HTLV-1 promueve la proliferación de estas células así como linfocitos CD8 y células NK (19) alterando la función de los linfocitos CD4 a través de una serie de factores de transcripción como la proteína TAX, FOXP3 y el factor nuclear de células $\mathrm{T}$ activadas (NFAT), con el desarrollo de respuesta inmune deficiente y autoinmunidad (20).

El presente caso pone de relieve la importancia de buscar una causa subyacente en la presencia de $\mathrm{SH}$ y aporta al rol inmunosupresor del virus de HTLV-1 en el desarrollo de histoplasmosis diseminada.

\section{Declaración de conflictos de intereses:}

Los autores declaran no tener conflictos de interés con ninguna institución pública ni privada.

\section{Contribución de autoría:}

RV concibió el reporte de caso, búsqueda bibliográfica y elaboración del reporte preliminar; DC realizó las pruebas para el diagnóstico de Histoplasmosis y ambos contribuyeron intelectualmente en la redacción del informe final.

\section{Correspondencia:}

Ronald Ventosilla

Correo electrónico: ronaldventosilla@yahoo.com

\section{REFERENCIAS BIBLIOGRÁFICAS}

1. Emmenegger U, Schaer DJ, Larrochec C, Neftel KA. Haemophagocytic syndromes in adults: current concepts and challenges ahead. Swiss Med Wkly. 2005; 135: 299-314.

2. Douka E, Economidou F, Nanas S. Infections associated with the hemophagocytic syndrome. Hospital Chronicles. 2012; 7(1): 16-24.

3. Weitzman S. Approach to Hemophagocytic Syndromes. ASH Education Book. 2011. pp: 178-183. Disponible en: http://asheducationbook. hematologylibrary.org/content/2011/1/178.full.pdf

4. Henter JI, Elinder G, Ost A. Diagnostic guidelines for hemophagocytic lymphohistiocytosis. Semin Oncol. 1991; 18:29-33.

5. Jordan MB, Allen CE, Weitzman S, Filipovich $\mathrm{AH}$, McClain KL. How I treat hemophagocytic lymphohistiocytosis. Blood. 2011; 118: 4041-52.

6. Tang $\mathrm{Y}, \mathrm{Xu}$ J. Advances in Hemophagocytic Lymphohistiocytosis: Pathogenesis, Early Diagnosis/ Differential Diagnosis, and Treatment. The Scientific World Journal. 2011; 11: 697-708.

7. Van Koeveringe MP, Brouwer RE. Histoplasma capsulatum reactivation with haemophagocytic syndrome in a patient with chronic lymphocytic leukaemia. The Journal of Medicine. 2010; 68(12): 418-21.

8. Sanchez A, Celaya AK, Victorio A. HistoplasmosisAssociated Hemophagocytic Syndrome: A case report. The AIDS Reader. 2007; 17(10): 1-6.

9. Grateau G, Bachmeyer C, Blanche $P$, et al. Haemophagocytic syndrome in patients infected with the human immunodeficiency virus: nine cases and a 
review. J Infect. 1997; 34: 219-225.

10. Huang AM. Hemophagocytic lymphohistiocytosis and disseminated histoplasmosis. Blood. 2014; 123: 2449.

11. Gil-Brusola A, Pemán J, Santos M, Salavert M, Lacruz J, Gobernado M. Disseminated histoplasmosis with hemophagocytic syndrome in a patient with AIDS: description of one case and review of the Spanish literature. Rev Iberoam Micol. 2007; 24: 312-316.

12. Bustamante B, Seas C, Sanchez J, Gottuzo E. Histoplasmosis diseminada en SIDA presentación de un caso y revisión de la literatura. Rev Med Hered. 1990; 1(1):1-3.

13. Soto L, Legua P, Gottuzo E, et al. Paciente Inmunosuprimido con fiebre y radiografía con patrón miliar: Reporte de un caso. Rev Med Hered. 2005; 16: 218-222.

14. Canelo C, Cuadra J, Atencia F, Romani F. Human T Lymphotropic virus-1 associated gastrointestinal histoplasmosis in Peru. Infect Dev Ctries. 2011; 5(6): 484-488.

15. Ita F, Mayer EF, Verdonck K, Gonzalez E, Clark D, Gotuzzo E. Human T-lymphotropic virus type 1 infection is frequent in rural communities of the southern Andes of Peru. International Journal of Infectious Diseases.2014; 19: 46-52.

16. Verdonck K, González E, Schrooten W, Vanham G,
Gotuzzo E. HTLV-1 infection is associated with a history of active tuberculosis among family members of HTLV-1-infected patients in Peru. Epidemiol Infect. 2008; 136: 1076-1083.

17. Verdonk K, Henriquez C, Echevarria J, et al. Asociacion entre infección por el virus linfotrópico humano de células T tipo 1 (HTLV-1) y mortalidad en pacientes hospitalizados con tuberculosis. Rev Med Hered. 2004; 15(4): 197-202.

18. León M, Alave J, Bustamante B, Gotuzzo E, Seas C. A probable association between HTLV-1 and endemic mycosis in Latin America. Letter to the editor J Infect Dev Ctries. 2012; 6(3):301.

19. Coutinho R, Rios MF, Korngold AB, Nilla V, GalvãoCastro B, Elizabeth R. Human T lymphotropic virus type 1 (HTLV-1) proviral load induces activation of T-lymphocytes in asymptomatic carriers. BMC Infectious Diseases. 2014; 14:453.

20. Quaresma JA, Yoshikawa GT, Koyama RB, Dias GA, Fujihara S, Fuzzi HT. HTLV-1, Immune response and autoimmunity. Viruses. 2016; 24(8): 1.

Recibido: 23/07/2015

Aceptado: 06/03/2015 